\title{
ASPECTOS DO DESIGN INSTRUCIONAL NA ADAPTAÇÃO DE CONTEÚDOS EDUCACIONAIS PARA APLICATIVOS M-LEARNING
}

\author{
SÃO LUÍS/MA AGOSTO/2018
}

\author{
Karoline Correa Trindade - UNASUS/UFMA - karolinecorreatrindade@gmail.com \\ Stephanie Matos Silva - UNASUS/UFMA - stephanie.matos01@gmail.com \\ Isabelle Aguiar Prado - UNASUS/UFMA - isabelleaguiarpp@gmail.com \\ Ludmila Gratz Melo - UFMA - ludgratz@gmail.com \\ Paola Trindade Garcia - UNASUS/UFMA - paolatrindadegarcia@gmail.com \\ Regimarina Soares Reis - UNASUS/UFMA - regimarina.reis@gmail.com \\ Ana Emília Figueiredo Oliveira - UNASUS/UFMA - oliveira.anaemilia@gmail.com
}

Tipo: Relato de Experiência Inovadora (EI)

Categoria: Métodos e Tecnologias

Setor Educacional: EDUCAÇÃO CONTINUADA EM GERAL

\begin{abstract}
RESUMO
O crescente desenvolvimento das tecnologias da informação e da comunicação (TICs), aliado à popularização do uso de dispositivos móveis em todo o mundo, tem potencializado o alcance da educação a distância no fornecimento de ferramentas de apoio ao processo de ensino-aprendizagem. Nesse contexto surge a proposta do m-learning, processo de ensino mediado por dispositivos móveis. A utilização deste tipo de ferramentas apresenta algumas vantagens, entre elas, a mobilidade e acesso ao conteúdo sem conexão de internet. No entanto, também cresce a preocupação com a eficácia dos materiais educacionais desenvolvidos para estes dispositivos. Neste ponto, destacam-se as estratégias desenvolvidas pelo design instrucional - DI. Focado na adaptação de conteúdos para ofertas educacionais, o DI tem se destacado na concepção de produtos voltados para a aprendizagem online. Este artigo objetiva relatar as estratégias de DI utilizadas pela equipe da UNA-SUS/UFMA nos e-books produzidos pela instituição.
\end{abstract}

Palavras-chave: m-learning; design instrucional; educação a distância 


\section{Introdução}

O crescente desenvolvimento das tecnologias da informação e da comunicação (TICs), aliado à popularização do uso de dispositivos móveis em todo o mundo, tem potencializado o alcance da educação a distância no fornecimento de ferramentas de apoio ao processo de ensino-aprendizagem. Juntamente com a expansão da internet, o grande alcance das telecomunicações móveis tem sido uma característica notável do desenvolvimento socioeconômico global. De acordo com as estatísticas da União Internacional de Telecomunicações, o número de assinaturas de celulares no mundo era próxima ao número habitantes do planeta Terra em 2014 - estimado em aproximadamente 7 bilhões de pessoas (UIT, 2015).

A facilidade de acesso à ferramentas educacionais por meio de aparelhos portáteis cada vez mais insere o conceito de mobilidade no planejamento de recursos educacionais. Derivado do e-Learning, o mobile learning ou m-learning é entendido como a junção dos conceitos de mobilidade e aprendizagem (OLIVEIRA et al., 2014). Assim, configura-se como o processo de ensino mediado por dispositivos móveis, tais como smartphones, tablets, micronotebooks, dentre outros (KEARNEY et al., 2012).

Segundo Saccol, Schlemmer e Barbosa (2011), m-learning pode ser definido como:

"processos de aprendizagem apoiados pelo uso de tecnologias da informação ou comunicação móveis e sem fio, cuja característica fundamental é a mobilidade dos aprendizes, que podem estar distante uns dos outros e também de espaços formais de educação, tais como salas de aula, salas de formação, capacitação e treinamento ou local de trabalho" (p. 23).

Para Georgiev, Georgieva e Smrikarov (2004) a definição de m-learning deve incluir também a habilidade de aprender em qualquer lugar, a todo momento e sem conexão de internet física permanente.

O mobile learning facilita a igualdade de oportunidades para todos, permitindo que a aprendizagem seja acessível em diferentes localizações, reduzindo os impactos da distância geográfica para o processo de ensino-aprendizagem. Os dispositivos móveis são pequenos e portáteis, o que permite aos alunos usá-los em qualquer hora e lugar para interagir com outros alunos, compartilhar informações e conhecimentos, completar uma tarefa ou trabalhar em colaboração em um projeto (ALLY \& PIETRO-BLÁZQUEZ, 2014).

O uso de dispositivos móveis possibilita a ampliação do uso das TICs para a aprendizagem, com acesso facilitado a recursos educacionais diferenciados 
(CARVALHO, 2012). Oliveira et al (2014) apontam algumas outras vantagens do uso de aplicativos móveis para uso na educação, como: autonomia, portabilidade/mobilidade, facilidade de entendimento e flexibilidade. Entretanto, as mudanças na tecnologia alteram as possibilidades de aprendizagem e criam novos desafios (ROSSING et al. 2012).

O design do m-learning é um empreendimento complexo que requer repensar o papel e as relações entre pedagogia, tecnologia e aprendizes (GEDIK et al, 2012). Nesse sentido, o designer instrucional deve, além de estabelecer a conexão com conteúdos, objetivos e estratégias instrucionais, oferecer recursos tecnológicos condizentes com a plataforma utilizada e que envolvam o estudante no processo de aprendizagem. Assim, apesar dos aspectos positivos, ainda são necessários estudos que possam discutir os fundamentos pedagógicos de materiais nestes formatos visando avaliar os alcances possibilitados por estas ferramentas de ensino nos tempos atuais. Nesse sentido, este artigo pretende descrever qual escopo de trabalho, desde a concepção até a elaboração final, do design instrucional para produção de materiais educacionais da UNASUS/UFMA para m-learning.

\section{Objetivos}

\subsection{Objetivo geral}

- Descrever as estratégias de design instrucional (DI) utilizadas pela equipe da UNA-SUS/UFMA na concepção de materiais educacionais para ofertas mlearning.

\subsection{Objetivos específicos}

- Discutir a inserção do designer instrucional na concepção de recursos educacionais para $\mathrm{EaD}$, com enfoque na aprendizagem com a utilização de dispositivos móveis.

- Apresentar as etapas do processo de construção das estratégias educacionais voltadas para mobile learning.

\section{0 papel do design instrucional na concepção de recursos educacionais}

O design instrucional de um curso a distância é formado a partir da associação das diversos componentes, como o planejamento didático, a concepção metodológica, texto base, o ambiente virtual, as atividades e avaliações. A correlação entre tais elementos é 
definida a partir da abordagem pedagógica, que orienta a concepção das etapas subsequentes, sendo idealizada no momento inicial da concepção de qualquer produto educacional (FRANÇA, 2007).

O designer instrucional é o profissional com perfil interdisciplinar, em especial nas áreas de educação, comunicação e tecnologia, articulando várias funções. Acompanha o processo desde o planejamento até a etapa de avaliação de um curso ou atividade a distância (MOREIRA, 2009). Segundo a definição de Souza (1999 apud in FRANCO, 2010. p. 89), planejar é:

"explicitar de forma articulada a justificativa, a caracterização do contexto, o perfil da clientela e os objetivos de um determinado projeto. Essa articulação definirá a metodologia e sua fundamentação teórica e que, em um plano detalhado, orientará e definirá os contornos para a elaboração do material didático".

Kenski (2015) afirma que o design instrucional (DI) está ligado à produção de soluções educacionais congruentes às necessidades de aprendizagem de determinado grupo de pessoas. Desta forma não se pode falar em DI sem considerar ações de planejamento visando a adaptação de conteúdos para ofertas em educação, especialmente online.

Conceituado por Filatro (2015) como "ação intencional e sistemática de ensino que envolve o planejamento, desenvolvimento e aplicação de métodos, técnicas, atividades, materiais, eventos e produtos educacionais em situações didáticas específicas, a fim de promover, a partir dos princípios de aprendizagem e instrução conhecidos, a aprendizagem humana", o produto de DI expressa concretamente os objetivos de uma produção educacional e as estratégias selecionadas para alcançá-los, conforme ressalta Kenski (2015). Por estas características, o DI tem sido peça fundamental na concepção de produtos voltados para o ensino online, especialmente diante do crescimento cada vez maior das demandas em educação a distância. A sistematização das etapas de aprendizagem em materiais planejados para as peculiaridades do ensino não presencial representam potencialidades para o futuro da educação mediada por tecnologias.

\section{Adaptação pedagógica de conteúdos para aplicativos m-learning}

A forma de apresentação do conteúdo na educação a distância sofreu inúmeras modificações ao longo dos anos, pela própria evolução das tecnologias digitais (VIDAL \& MAIA, 2010). As peculiaridades inseridas pela utilização de dispositivos móveis como ferramentas de ensino tem desafiado o planejamento de materiais adaptados à estas novas necessidades. A condução autônoma do aluno no material didático exige novos modos de conceber o ensino à distância sob o viés da portabilidade. 
Para Renato Bulcão (2009) a educação a distância mediada por dispositivos móveis na veiculação e promoção educativa é uma possibilidade tecnológica que traz a realização de atividades pedagógicas em diferentes espaços e meios independentes da localização física e/ou espacial dos sujeitos na realização de cursos. O conceito de aprendizagem autodirigida, de acordo com Palange (2015), refere a um modelo no qual o próprio aluno planeja, desenvolve e regula a aprendizagem usando os recursos que se encontram disponíveis. Desta maneira, o DI de materiais para aplicativos m-learning deve considerar as possibilidades de interação do aluno ao acessar o conteúdo educacional.

Marçal et al. (2005, p. 3) afirma que:

[...] o m-learning surge como uma importante alternativa de ensino a distância, na qual podem ser destacados os seguintes objetivos: melhorar os recursos para o aprendizado do aluno, que poderá contar com um dispositivo computacional para execução de tarefas (...); prover acesso aos conteúdos didáticos em qualquer lugar e a qualquer momento(...); aumentar as possibilidades de acesso ao conteúdo, incrementando e incentivando a utilização dos serviços providos pela instituição, educacional ou empresarial; expandir o corpo de professores e as estratégias de aprendizado disponíveis, através de novas tecnologias que dão suporte tanto à aprendizagem formal como à informal; expandir os limites internos e externos da sala de aula ou da empresa, de forma ubíqua; fornecer meios para o desenvolvimento de métodos inovadores de ensino e de treinamento, utilizando os novos recursos de computação e de mobilidade.

É possível observar que há uma extensa gama de atividades relacionadas ao conceito de m-learning. Nessa perspectiva, não podemos considerar o m-learning apenas como uma outra apresentação do e-learning, visto que os dispositivos móveis trazem possibilidades para além do ensino à distância "tradicional", diferenciando o m-learning de ofertas educacionais (SACCOL; SCHLEMMER; BARBOSA, 2011).

Interatividade, mobilidade, prática de trabalho em equipe, aprendizagens em contextos reais, são características do m-learning que têm motivado pesquisas sobre o uso de dispositivos móveis na educação (SHARPLES et al., 2009; ALLY, 2009; RUCHTER et al., 2010). Tal fato é fato reiterado por Ferriman (2013), o qual afirma que o m-learning poderá ser um dos principais métodos de aprendizado no futuro.

\section{Produção de recursos educacionais para m-learning na UNA-SUS/UFMA}

Desde 2011 a UNA-SUS/UFMA tem ofertado cursos no modelo MOOC destinados a profissionais da saúde integradas ao sistema público de saúde brasileiro. A escolha pelas temáticas disponibilizadas parte de diagnósticos de necessidades identificadas especialmente pelo Ministério da Saúde, o qual demanda os temas que necessitam serem abordados, sendo uma das formas de manter a qualidade dos serviços de saúde fornecidos à população. 
O aprendizado projetado para tecnologias móveis e sem fio oferece uma perspectiva que difere da concepção de e-learning para computadores. Diante das peculiaridades do m-learning, fez-se necessário a proposição de um modelo eficiente para o planejamento destes materiais que fosse capaz de conciliar as barreiras pela distância com os objetivos educacionais traçados para os cursos.

A educação mediada por dispositivos móveis portáteis possibilita que o processo de ensino-aprendizado ocorra em diferentes ambientes. Neste sentido, os recursos educacionais devem ser produzidos na perspectiva de permitir ao usuário liberdade neste processo. No contexto da UNA-SUS/UFMA, os e-books produzidos para mlearning são disponibilizados online e offline, alocados em uma loja virtual gratuita, a SAITE STORE, permitindo acesso facilitado do usuário aos conteúdos educacionais, com navegação responsiva e intuitiva, permitindo ao usuário a montagem de sua própria biblioteca de e-books (SAITE/STORE, 2018).

Os fundamentos teóricos preconizados pelo design instrucional, nesta perspectiva, colaboraram para esta finalidade fornecendo background capaz de sustentar as decisões pedagógicas congruentes com as diretrizes pactuadas no plano didático. A concepção de projetos em DI prevê a existência de etapas que norteiam a elaboração até a oferta ao público-alvo, sendo o material final a expressão de todo o processo anterior de planejamento.

A concepção de projetos em DI prevê a existência de etapas que norteiam a elaboração até a oferta ao público-alvo, sendo o material final a expressão de todo o processo anterior de criação e planejamento. Inspirando-se no modelo proposto por Filatro e Cairo (2015), a adaptação de materiais educacionais na UNA-SUS/UFMA para m-learning considera as fases: análise diagnóstica, transposição didática, elaboração de briefings e validação do protótipo online.

A análise diagnóstica envolve, para além da identificação de informações gerais sobre a oferta trabalhada e tipologia do curso, o detalhamento de perfil do público alvo. Algumas questões norteadoras são levantadas nessa fase: trata-se de curso autoinstrucional? Trata-se de um curso instrucional? De um curso de especialização? De um curso de capacitação e/ou extensão? Exige-se a avaliação do nível de habituação dos usuários ao uso destas plataformas para fins educacionais. Este é um aspecto a ser considerado, pois dependendo dos perfis, as gradações de acessibilidade ao uso de tecnologias são diversas e podem interferir no manuseio do aplicativo.

Ainda na fase de análise diagnóstica, identificam-se os objetivos educacionais, etapa 
fundamental para direcionar as propostas pedagógicas e metodológicas a serem implantadas pelo design instrucional, a fim de respeitar o planejamento educacional do curso proposto. São definidas também as estratégias educacionais para aproximar o aluno de situações práticas reais, como as situações-problemas. Como produtos da etapa de análise diagnóstica tem-se a organização de mapas mentais, com os principais tópicos abordados e suas respectivas estratégias pedagógicas.

A transposição didática refere-se à primeira etapa prática da concepção, pois consiste no momento em que as estratégias pedagógicas são montadas, estruturadas e aplicadas no mockup. A leitura exploratória do texto da unidade de ensino possibilita a imersão no conteúdo abordado e potencializa a proposição de objetos educacionais coerentes com o objetivo da unidade de ensino. Na transposição os conteúdos são reestruturados visando melhor adequação ao aplicativo. Uma aspecto importante a ser considerado é a adaptabilidade dos recursos às diferentes dimensões de tela. Por vezes, o espaço reduzido é quesito relevante a ser pensado na proposição. Da mesma forma, os cliques e tamanho das imagens precisam ser ponderados para que a navegabilidade do material não seja prejudicada.

A estruturação dos conteúdos abordados deve valorizar o encadeamento lógico, hierarquizando-se os níveis de relevância para o alcance do objetivo educacional. Assim, a interatividade deve ser pensada como forma de potencializar o acesso e a autonomia do aluno ao tema tratado. Incorre-se, muitas vezes, na secundarização de conteúdos que podem prejudicar o melhor aproveitamento do recurso. A figura 1 demonstra a adaptação pedagógica de fluxograma para utilização em m-learning.

Figura 1. Imagem de livro multimídia em aplicativo educacional sobre cuidados odontológicos de paciente com hipertensão (UNA-SUS/UFMA - USP, 2017).

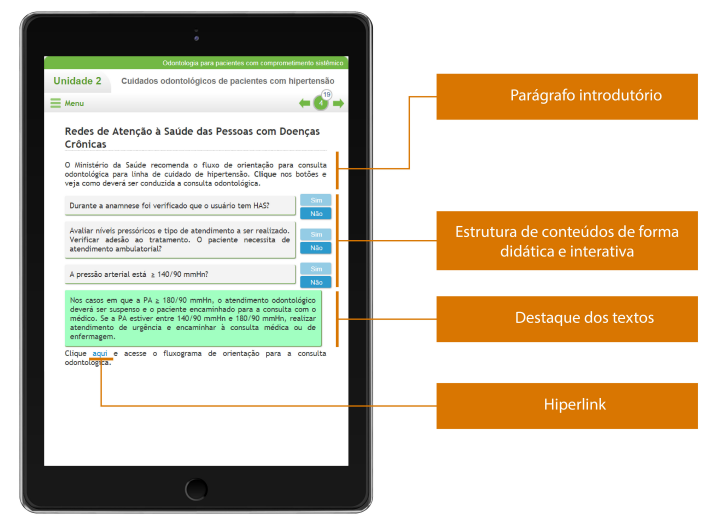

A terceira etapa de concepção do design instrucional consiste na elaboração de briefings. Para cada recurso idealizado na composição didática elaboram-se estes 
materiais norteadores para criação de objetos de aprendizagens ou ilustrações que irão compor o e-book, dentre as quais as mais frequentes são as equipes de design gráfico e de tecnologia da informação. O briefing refere-se a um documento contendo a descrição para composição de elementos didáticos. A partir da concepção do briefing é possível diálogo mais efetivo entre as equipes intersetoriais para elaboração dos recursos de maneira a associar as questões pedagógicas e técnicas às tecnológicas, em prol do aprendizado, considerando-se a aplicação em dispositivos móveis. A escolha de cores, fontes, tamanhos e estilos de ilustrações requer critérios diferenciados devido às dimensões da tela dos dispositivos móveis, bem como resolução e características de cada aparelho.

Por fim, ocorre a validação do protótipo após programação do e-book. Nesse momento que é realizada a verificação tanto da adequação dos recursos educacionais aos objetivos do material, bem como a checagem das funcionalidades aplicadas. É feita uma avaliação por parte dos desenvolvedores envolvidos, como designers gráficos, designers instrucionais, programadores e equipe pedagógica para a chancela do e-book final. Em alguns casos, novas propostas devem são elaboradas na identificação de recursos não funcionais ou aplicações que não mostraram-se efetivas.

Vale ressaltar que nem nesta, nem nas etapas anteriores, o processo de construção, revisão ou validação de produtos é realizado de forma estanque: trata-se de um processo coletivo, construtivo e dinâmico.

\section{Considerações finais}

Conforme pontuado por Saccol (2011), o m-learning propõe um ensino a distância mais dinâmico e interativo por meio da inclusão dos dispositivos móveis ou portáteis (smartphones e tablets) na elaboração de atividades, sem que os limites físicos e a inércia de cabos comprometam seu uso. A difusão do m-learning aponta para a necessidade de adequação dos processos de planejamento educacional. Nesse contexto, a figura do designer instrucional torna-se imprescindível para que sejam propostos materiais ricos e atrativos, capazes de desenvolver novas habilidades cognitivas no aprendiz.

\section{Referências}

ALLY, M; PRIETO-BLÁZQUEZ, J. What is the future of mobile learning in education?. International Journal of Educational Technology in Higher Education, v. 11, n. 1, p. 142-151, 2014. 
ALLY, M. Mobile learning: Transforming the delivery of education and training. Athabasca University Press, 2009.

BULCÃO, R. Aprendizagem por m-learning. In: FORMIGA, M e LITTO, F M. Educação a distância: O estado da arte. São Paulo: Pearson Education, 2009.

CARVALHO, V. M. M. G de. Expectativas dos estudantes adultos do ensino superior a distância sobre a utilização de dispositivos móveis para a aprendizagem. 2012. Dissertação (Mestrado) - Universidade Aberta, Lisboa, 2012

FILATRO, A; CAIRO, Sabrina. Produção de conteúdos educacionais. São Paulo: Saraiva, 2015.

FERRIMAN, J. Mobile Learning On The Rise. 2013. Disponível em:. http://www.learndash.com/mobile-learning-on-the-rise-infographic. Acesso em: 21 mar. 2018.

FRANÇA, G. O Design Instrucional na Educação a Distância. São Paulo: Editora Esfera, 2007.

FRANCO, L. R. H. R. EaD Virtual: entre a teoria e a prática. Itajubá: Ed. Premier, UNIFEI, 2010

GEORGIEV, T; GEORGIEVA, E; SMRIKAROV, A. M-learning-a New Stage of ?Learning. In: International conference on computer systems and technologiesCompSysTech. 2004. p. 1-4.

KEARNEY, M. et al. Viewing mobile learning from a pedagogical perspective. Research in learning technology, v. 20, 2012.

KENSKI, V. M. Design Instrucional para cursos on-line. São Paulo/SP: Editora Senac, 2015.

MARÇAL, E; SANTOS, R; VIDAL, C; ANDRADE, R; RIOS, R. MuseuM: Uma Aplicação de m-learning com Realidade Virtual. Seminário Integrado de Software e Hardware. São Leopoldo: Unisinos, 2005.

MOREIRA, M. G. A composição e o funcionamento da equipe de produção In: Educação a Distância: o estado da arte / Fredric Michael Litto, Manuel Marcos Maciel 
Formiga (orgs). São Paulo: Pearson Education do Brasil, 2009.

OLIVEIRA, E. D. S. et al. Proposta de um modelo de cursos baseado em mobile learning: um experimento com professores e tutores no Whatsapp. In: XI ESUD Congresso Brasileiro de Ensino Superior a Distância, Florianópolis-SC. Pesquisa na EaD: reflexões sobre teoria e prática. Florianópolis-SC: NUTE UFSC. 2014. p. 3482-3496.

PALANGE, I. Produção de design instrucional para EAD: aprendizagem autodirigida, aprendizagem colaborativa, conectivismo e modelo ADDIE. In: KENSKI, V. M. Design Instrucional para cursos on-line. São Paulo: Editora Senac, 2015.

ROSSING, J. P. et al. ILearning: the future of higher education: students perceptions on learning with mobile tablets. Journal of the Scholarship and Teaching and Learning, p. 1-26, jun. 2012.

RUCHTER, M.; KLAR, B.; GEIGER, W. Comparing the effects of mobile computers and traditional approaches in environmental education. Computers \& Education, Oxford, UK: Elsevier Scienc Ltd, v. 54, p. 1054-1067, 2010.

SAITE STORE. Loja Virtual de E-books Interativos e Gratuitos. Disponível em: http:/www.saitestore.com.br/?page_id=2\&no-refresh=true\&lang=pt. Acesso em: 07/05/18.

SACCOL, A.; SCHLEMMER, E.; BARBOSA, J.. M-learning e u-learning: novas perspectivas de aprendizagem móvel e ubíqua. São Paulo: Pearson Prentice Hall, 2011.

SHARPLES, M.; ARNEDILLO-SÁNCHEZ, I.; MILRAD, M.: and VALVOULA, G. Mobile Learning: small devices, big issues. In: Balacheff, N., Ludvigsen, S., Jong, T., Lazonder, A. and Barnes, S. (Ed.). Technology-Enhanced Learning: principles and products. Netherlands: Springer. p. 233-249, 2009.

VIDAL, E. M.; MAIA, J. E. B. Introdução à Educação a Distância. RDS Editora, 2010.

UIT. M-powering development initiative: a report by the m-powering development initiative advisory board. Switzerland, Geneva, 2015. 